\title{
Application of vectorcardiography and recurrence-based methods to analysis of ECG signals
}

\author{
Joanna Iwaniec $^{1, *}$, Marek Iwaniec $^{2}$ and Antoni Kalukiewicz ${ }^{3}$ \\ ${ }^{1}$ AGH University of Science and Technology, Faculty of Mechanical Engineering and Robotics, Department of Rob otics and \\ Mechatronics, Mickiewicz Alley 30, 30-059 Kraków, Poland \\ ${ }^{2}$ AGH University of Science and Technology, Faculty of Mechanical Engineering and Robotics, Department of Process Control, \\ Mickiewicz Alley 30, 30-059 Kraków, Poland \\ ${ }^{3}$ AGH University of Science and Technology, Faculty of Mechanical Engineering and Robotics, Department of Mining, \\ Dressing and Transport Machines, Mickiewicz Alley 30, 30-059 Kraków, Poland
}

\begin{abstract}
The paper concerns application of vectorcardiography and recurrence-based methods to analyze ECG signals. The first method involves recording the direction and magnitude of electrical forces of the heart by means of a continuous series of vectors that form a curving line around a center, while the second consists in representing the ECG signals in the phase space and searching for the recurrence properties of the registered signals. In both methods, change of analysis domain offers new opportunities for early diagnosis. In this work, discussed methods have been used for the purposes of analysis of selected ECG signals stored in the medical database. Vectorcardiograms were determined on the basis of ECG signals for the Farnk's leads. Signal analysis was carried out with the application of the recurrence plots (RP) method. The proposed approach simplifies assessment of the signal information content by providing patterns characteristic for different myocardial injury. Carried out research requires complementation by results of statistical analysis for various age groups and degrees of myocardial injury.
\end{abstract}

\section{Introduction}

For many years, by far the greatest threat to the health and life all over the World have been diseases of the circulatory system. The basic and the most efficient tool used in cardiovascular diagnostic is ECG, which is so widespread that it is difficult to determine the exact number of these tests performed each day. Despite the requirement for the efficient and reliable methods of ECG signals analysis and interpretation, in practice, almost exclusively, classical methods involving analysis of geometrical properties of the recorded waveforms in time domain are used. The scientific works carried out recently have been focused on increasing the accuracy of ECG analysis by increasing the accuracy of finding its characteristic points. However, the accuracy limits of such a geometrical analysis have already been reached. Therefore the quest of formulating novel methods supporting classical analysis of ECG signals is still open.

In this work, in order to support classical methods of ECG analysis, vectorcardiography and recurrence-based methods have been used. The first method involves recording the direction and magnitude of electrical forces of the heart by means of a continuous series of vectors that form a curving line around a centre. The main idea of the second method consists in representing the ECG signals in the phase space and searching for the recurrence properties of the registered signals.
In both methods, change of analysis domain offers new, so far unreachable, opportunities for early diagnosis. In this work, discussed methods have been used for the purposes of analysis of selected ECG signals stored in the medical database The PTB Diagnostic ECG Database. Patients were selected from the group of male smokers after the age of 50. Vectorcardiograms were determined on the basis of ECG signals for Farnk's leads. The further analysis was carried out with the application of the recurrence plots (RP) and RQA analysis methods.

The structure of the paper is as follows. Section 2 concerns electrocardiography and vectorcardiography. Recurrence-based methods are discussed in the section 3. In the section 4 selected experimental results are presented. Finally, the paper is concluded in section 5 .

\section{Vectocardiography}

Currently, electrocardiography is one of the most extensively described procedures of modern medicine. Ease of implementation, low costs and a relatively high diagnostic value make it a tool useful for every clinician. The electrodes on the patient's body are positioned in such a way that they project the heart work on two planes - the front one (three limb leads - aVR, aVL, $\mathrm{aVF}$ ) and transverse (six precordial leads, from $\mathrm{V}_{1}$ to $\mathrm{V}_{6}$ ). In addition, it is possible to extend the set of electrodes to revise cardiac areas that are not available in

Corresponding author: jiwaniec@agh.edu.pl 
the routine testing. Typical result of the test has the form of a printout of voltage in the function of time for all the leads mentioned above, additionally extended by bipolar leads, which are called differential leads since they register differences between selected unipolar leads. Lead I presents the difference between aVL and aVR, lead II - between aVF and aVR while III - between aVF and aVL. They do not add any new information to the electrocardiogram data space, but only improve its interpretation by the clinician. A lot of basic diagnostic information can be derived based on the signal from lead II only.

Vectorcardiography [1] presents slightly different approach. Since the working heart behaves like a dipole, it is possible to analyse changes of a dipole moment in time. The idea of the method consists in conversion of one-dimensional information from each lead into three coordinates in the Cartesian coordinate system, which reduces the dimensions of the data space from originally nine to three. Visualization of a vectorcardiogram is usually performed using specialistic software. It has the form of a curve determined by a time-wandering vector of the dipole moment of the heart. The method is very promising since it shows the changes of direction and magnitude of the electrical forces of the heart $[2,3]$. This information is of key importance for the diagnosis of acute coronary syndromes. Unfortunately, in clinical practice, classical methods are still preferred. The hope for practical applications of vectorcardiography lies in the usage of numerical methods.

\section{Recurrence-based methods}

The formal concept of recurrences, in the sense of returning to the former states, was introduced by Henri Poincaré [4] in 1890. In 1987, based on the Poincaré's achievements, Eckmann, Oliffson and Ruelle formulated the Recurrence Plots (RP) method [5]. The method aims at revealing all the times when the phase space trajectory of the considered dynamical system visits roughly the same area in the phase space. Recurrence of given state $x$ (observed at time $i$ ) at a different time $j$ is marked with ones and zeros within so called recurrence matrix $[R]$ :

$$
\left[R_{i, j}\right]=\Theta\left(\varepsilon-\left\|\left\{x_{i}\right\}-\left\{x_{j}\right\}\right\|\right), i, j=1, \mathrm{~K}, N
$$

where $N$ denotes the number of considered states $x_{i}, \varepsilon_{i}$ threshold distance, \|\| a norm and $\Theta($ ) the Heaviside function.

Graphical representation of matrix $[R]$ is known under the term recurrence plot. In other words, the recurrence plot represents graphically the matrix $\left[R_{i, j}\right]$ given as:

$$
\left[R_{i, j}\right]=\left\{\begin{array}{ll}
1: & \left\{x_{i}\right\} \approx\left\{x_{j}\right. \\
0: & \left\{x_{i}\right\} \neq\left\{x_{j}\right.
\end{array}\right\} \quad i, j=1, \mathrm{~K}, N
$$

where $\left\{x_{i}\right\} \approx\left\{x_{j}\right\}$ are the points belonging to the neighbourhood of radius $\varepsilon$ (defined according to the applied norm).
In order to estimate recurrence plot for the given dynamical system it is necessary to specify values of three parameters: threshold $\varepsilon$, time delay $\tau$ and embedding dimension $m$. Values of these parameters have a significant influence on the informative content of the estimated recurrence plot and, therefore, should be selected carefully.

In the literature various criterions for selection of threshold $\varepsilon$ have been proposed $[6,7,8]$. The most commonly used criterion is the fixed amount of nearest neighbours (FAN) criterion, which consists in fixing the number of neighbours for each point of the trajectory [9]. In this case, the threshold is different for each point of the trajectory. The advantage of the method is that it preserves the recurrence point density and allows to compare RPs of different systems without the necessity of normalising the time series beforehand.

Appropriate time delay $\tau$ can be determined with the application of the auto-correlation or mutual information function $[10,11]$. For the purposes of the smallest sufficient embedding dimension $m$ estimation, the false nearest neighbours method [10] is frequently used. Originally, the RP method was used for the purposes of visualisation of system trajectories. Later, development of the Recurrence Quantification Analysis (RQA) [12], supporting interpretation of recurrence plots, has consolidated the RP method as a tool for nonlinear data analysis.

\section{Results of the experimental research}

Results of the research carried out over the last few years $[14,15,16]$ have proven that the recurrence-based methods are particularly sensitive to changes in the dynamic behaviour of mechanical and biological systems. In this paper, the methodology based on RPs method was applied to analysis of selected ECG signals stored in the medical database The PTB Diagnostic ECG Database. The database contains 549 records from 290 subjects (patients). Each subject is represented by one to five records. Each record includes 15 simultaneously measured signals: the conventional 12 leads together with the 3 Frank lead ECGs $(v x, v y, v z)$. Each signal is digitized at 1000 samples per second, with 16 bit resolution over a range of $\pm 16.384 \mathrm{mV}$.

In this paper, the proposed methodology is presented at the example of ECG signal of 66-year-old patient (patient no. 9 in the Database), performed 11 days after the infarction of the lower-lateral wall.

Patient vectorcardiograms are presented in the Fig. 1. In the ECG recording (Fig. 2) the most important changes are $\mathrm{ST}$ segment elevation in leads $\mathrm{V}_{2}, \mathrm{~V}_{3}, \mathrm{~V}_{4}$, negative $\mathrm{T}$ wave visible in lead $V_{6}$ and pathological $Q$ wave in leads II and III, which is characteristic for the infarction of the lower-lateral wall.

In the next step of analysis, ECG signals from Frank leads $(v x, v y, v z)$ were analysed by means of the recurrence plot method (Fig. 3). Minimal dimensions of the phase space $m$ were determined with the application of the false nearest neighbours criterion. In order to establish time delay $\tau$ mutual information function 
method was used. For the purposes of consistency, for all the analysed signals the same value of threshold ( $\varepsilon=0.1)$ was assumed. All the computations were carried out in the CRP Toolbox for MATLAB.
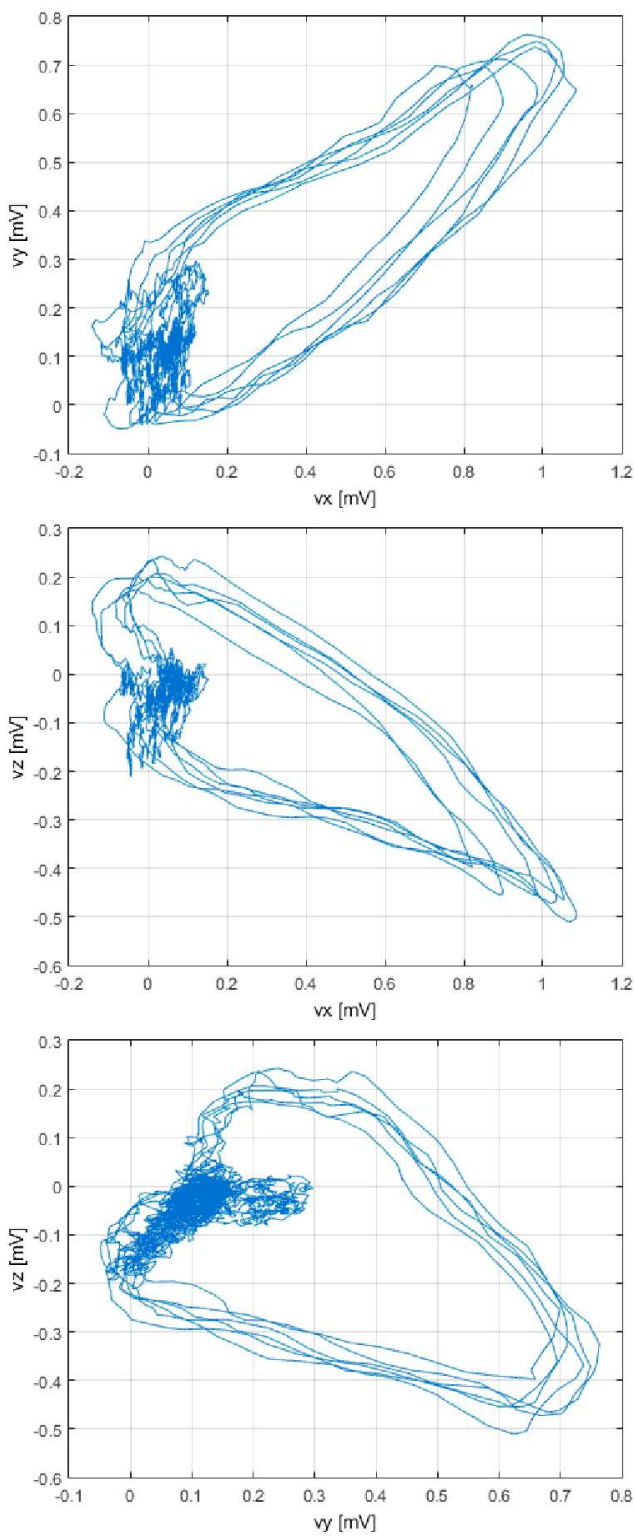

Fig. 1. Vectorcardiogram of the considered patient.

In the next step of analysis, conversion of analysed ECG signals from cartesian to spherical coordinates was performed:

$$
r=\sqrt{x^{2}+y^{2}+z^{2}}, \quad \phi=\operatorname{arctg} \frac{y}{x}, \quad \theta=\arcsin \frac{z}{r}
$$

Recurrence plots illustrating recurrent properties of the analysed signals in the spherical coordinates are presented in the Fig. 4.

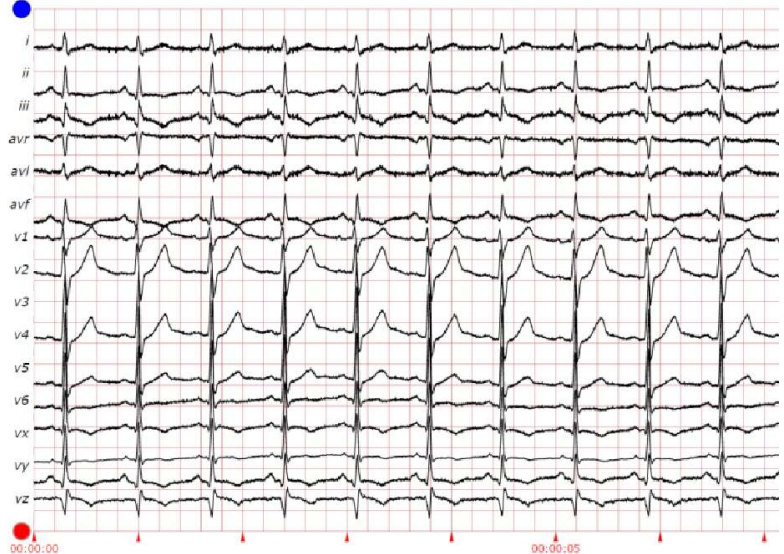

Fig. 2. ECG recording of the considered patient.

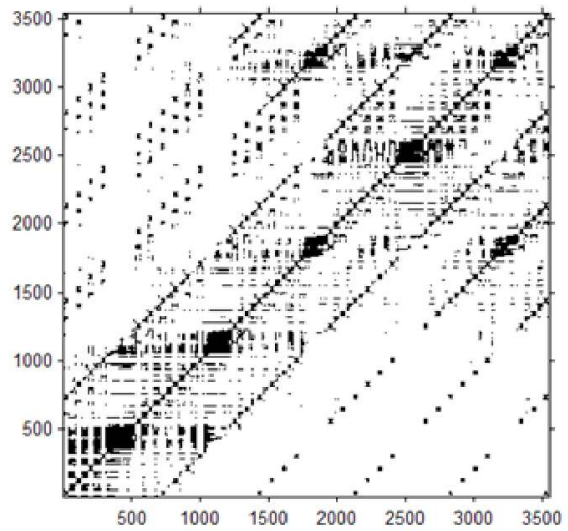

a) vx: $\tau=90, m=6, \varepsilon=0.1$, FAN

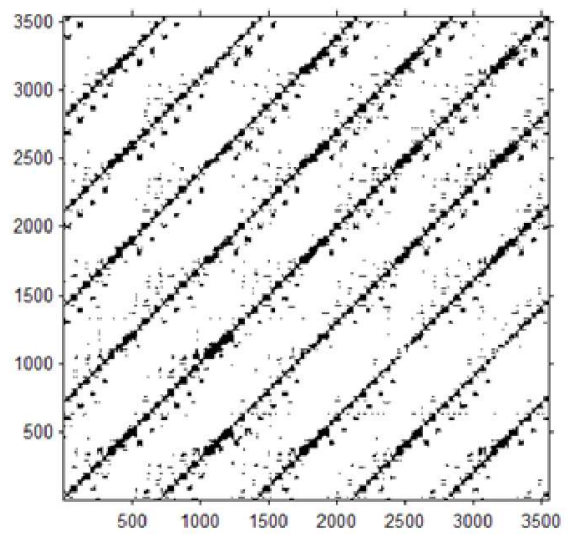

b) $\quad$ y: $\tau=90, m=6, \varepsilon=0.1, \mathrm{FAN}$

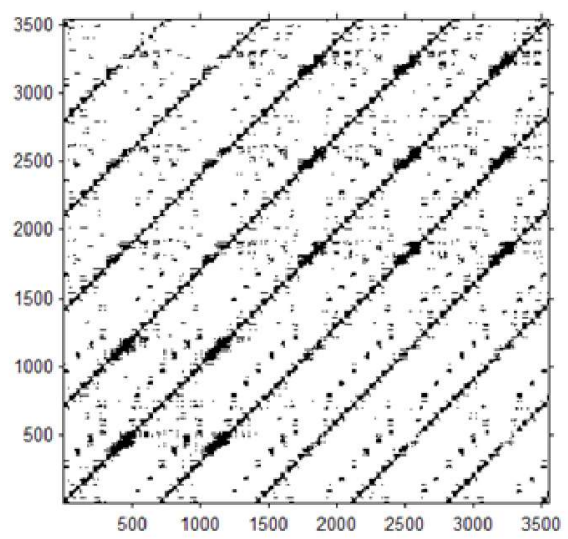

c) vz: $\tau=90, m=6, \varepsilon=0.1$, FAN

Fig. 3. RPs of ECG signals from Frank leads. 


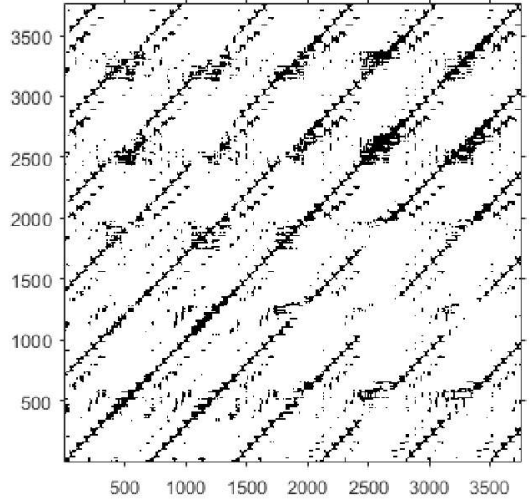

a) $\quad \operatorname{crp}(r): m=5, \tau=60, \varepsilon=0.1$, FAN

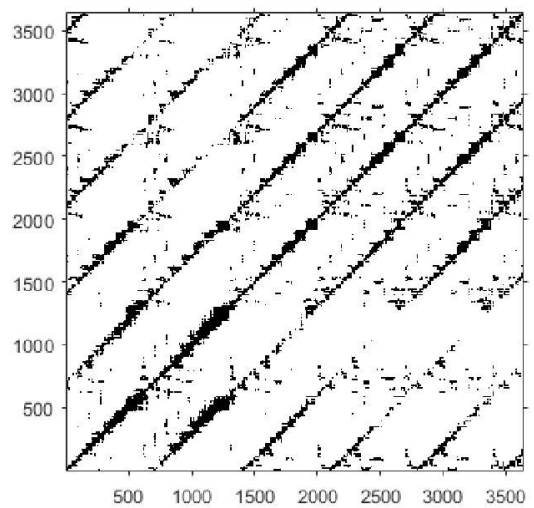

b) $\operatorname{crp}(\boldsymbol{\theta}): m=5, \tau=90, \varepsilon=0.1, \mathrm{FAN}$

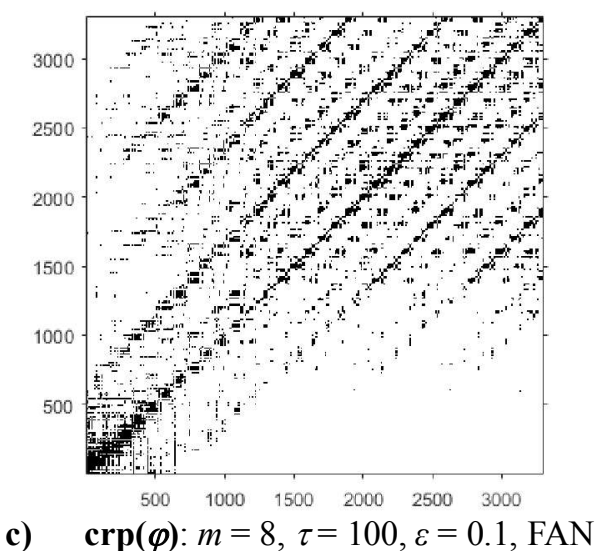

Fig. 4. RPs of ECG signals from Frank leads represented in the spherical coordinates.

The proposed approach simplifies assessment of the signal information content by providing patterns characteristic for different cardiovascular dysfunctions.

\section{Conclusions and final remarks}

In order to support classical methods of ECG signal interpretation in time domain, vectorcardiography and recurrence plots method were used. The essence of vectorcardiography consists in reduction of dimensions of the data space from originally nine (one-dimensional information from nine Einthoven leads) to three (three coordinates in the Cartesian coordinate system). The advantage of the method is the ability to observe the changes in the cardiac rhythm, which is of key importance in the diagnosis of acute coronary syndromes. However, such representation is difficult to interpret for cardiologists. Therefore, for the purposes of simplifying interpretation of information carried by the ECG signals from Frank leads $(v x, v y, v z)$ application of the recurrence plots method was proposed. Selection of RP method is natural due to the cyclic character of the heart work

Conducted studies have to be continued and complemented by statistical analysis for different age groups and various degrees of severity of dysfunctions. However, at the present stage of the research, it can be concluded that application of the recurrence based methods can be successfully used for ECG rhythm analysis and for interpreting the results of vectorcardiography.

\section{References}

1. E. Frank, An Accurate, Clinically Practical System For Spatial Vectorcardiography, Circulation 13 (1956), 737 749.

2. G.E. Burch, The history of vectorcardiography, Med. His. Sup. 5 (1985), 103-131.

3. G. Daniel, G. Lissa, D. Medina Redondo, L. Vásquez, D. Zapata, Real-time 3D vectorcardiography: An application for didactic use, J. Phys. Conf. Ser. 90 (2007), doi:10.1088/1742-6596/90/1/012013.

4. H. Poincaré, Sur la probleme des trios corps et les equations de la dynamique, Acta Math. 13 (1890), 1-271.

5. J.P. Eckmann, K.S. Oliffson, D. Ruelle, Recurrence plots of dynamical systems, Europhys. Lett. 5 (1987), 973-977.

6. M. Thiel, M.C. Romano, J. Kurths, R. Meucci, E. Allaria, F.T. Arecchi, Influence of observational noise on the recurrence quantification analysis, Physica D 171, 3 (2002), 138-152.

7. N. Thomasson, T.J. Hoeppner, C.L. Webber, J.P. Zbilut, Recurrence quantification in epileptic EEGs, Phys. Lett. A 279, 1-2 (2001), 94-101.

8. J.P. Zbilut, C.L. Weber, Embedding and delays as derived from quantification of Recurrence Plot, Phys. Lett. A 171 (1992), 199-203.

9. N. Marwan, M.C. Romano, M. Thiel, J. Kurths, Recurrence plots for the analysis of complex systems, Phys. Rep. 438 (2007), 237-329.

10. H. Kantz, T. Schreiber, Nonlinear Time Series Analysis (University Press, Cambridge, 1997).

11. N. Marwan, Cross Recurrence Plot Toolbox for Matlab. Reference Manual. Version 5.15. Release 28.6 (available at: http://tocsy.pik-potsdam.de, 2010, accessed: 10 January 2015).

12. J.P. Zbilut, C.L. Weber, Embedding and delays as derived from quantification of Recurrence Plot, Phys. Lett. A 171 (1992), 199-203.

13. E.J. Ngamga, A. Nandi, R. Ramaswamy, M.C. Romano, M. Thiel, J. Kurths, Recurrences of strange attractors. PRAMANA-J. Phys. 70, 6 (2008), 1039-1045.

14. J. Iwaniec, P. Kurowski, Experimental verification of selected methods sensitivity to damage size and location, J. Vib. Control 23, 7 (2017), 1133-1151.

15. J. Iwaniec, Investigation of selected mechanical systems by recurrence plots method, Int. J. Struct. Stab. Dy. 13, 7 (2013).

16. J. Iwaniec, M. Iwaniec, Heart work analysis by means of recurrence-based methods, Diagnostyka 18, 4 (2017), 8996. 